\title{
A Scheduling Strategy of Light Supplement in Agricultural Greenhouse Based on the Displacement of Light Source Group
}

\author{
Baichen Zeng, Jie Lv, Mengyue Li \\ NARI School of Electrical and Automation Engineering, Nanjing Normal University, Nanjing, China \\ Email: 181835002@stu.njnu.edu.cn
}

How to cite this paper: Zeng, B.C., Lv, J. and Li, M.Y. (2020) A Scheduling Strategy of Light Supplement in Agricultural Greenhouse Based on the Displacement of Light Source Group. Journal of Applied Mathematics and Physics, 8, 1646-1653. https://doi.org/10.4236/jamp.2020.88125

Received: July 31, 2020

Accepted: August 23, 2020

Published: August 26, 2020

Copyright $\odot 2020$ by author(s) and Scientific Research Publishing Inc. This work is licensed under the Creative Commons Attribution International License (CC BY 4.0).

http://creativecommons.org/licenses/by/4.0/

\begin{abstract}
LED can effectively promote the growth of crops and improve the yield of crops. In order to make the crops grow evenly in the agricultural greenhouse, the uniformity of illumination is very important. Because of the importance of illumination uniformity to the growth of crops, this paper intends to establish the illuminance model of light source without considering the influence factors such as the difference of each light source group and air scattering. On this basis, the reasonable layout of each light source position in the light source group is considered. Therefore, a light replenishment scheduling strategy based on the displacement of light source group is proposed in this paper Improve the uniformity of illumination in agricultural greenhouse and reduce its operation cost. Experiments show that the strategy is effective.
\end{abstract}

\section{Keywords}

Supplementary Light, Illumination Uniformity, Light Source Group, Scheduling Strategy

\section{Introduction}

Agricultural crops mainly rely on natural light source for photosynthesis and growth. For the effect of sunlight, artificial lighting can be considered to enhance, which will accelerate the maturity of crops. Artificial light supplement for crops is not a new thing in recent years. Xenon lamp, fluorescent lamp and high pressure sodium lamp are usually used in traditional plant supplementary lighting. However, the efficiency of these lighting methods is still not high. In addition, plants growing with supplementary light or plants planted with artificial light need a great deal of power consumption. Excessive light radiation or light 
radiation that cannot be used by plants is a waste of energy, which will lead to an increase in cost [1].

With the rapid development of LED lighting technology, LED lighting is more energy-saving than traditional light sources. The application of LED lighting in agriculture can effectively reduce the waste of energy. In addition, LED can adjust different colors and spectra, and can flexibly adjust LED light source to accelerate the production speed of plants according to the different needs of crops in different periods Land use of energy [2] [3].

In order to ensure that the crops in the whole farm can receive the artificial light evenly, so as to ensure that the crops can be in roughly the same growth stage, this will be conducive to the selection of LED light source color and spectrum.

In this paper, the distribution uniformity (DU) of supplementary illumination of agricultural crops was studied in order to achieve a more reasonable distribution of light sources. Firstly, the illuminance distribution diagram of the light source is established, and the illumination range and intensity of each light source are analyzed, and the relevant mathematical model is established; then, a model is established in the existing light source mathematical model to determine how to arrange the light source group for each re installation. This model leads to a simulation of lighting system test; the uniformity of illumination distribution (DU) and the execution efficiency of different work schedules are estimated. In this stage, $\mathrm{Du}$ is the most important factor; finally, considering the lighting time, a more appropriate crop light compensation strategy is established to avoid the waste of light source, thus reducing the operating cost of the lighting system. Finally, the experimental results show that the proposed compensation strategy can improve the uniformity of supplementary illumination.

\section{The Development of Math Models}

This section intends to establish the mathematical model of light source and light source group. Our goal is to determine the number of light sources and the distance between them, and to find a work schedule for moving light sources, which also includes a list of where to move the pipes.

\subsection{Establishment of Light Source Illumination Model}

Light intensity is a physical term for the luminous flux of visible light received per unit area. Light intensity has a great influence on the photosynthesis of organisms. It can be measured by illuminometer. The light intensity of a single LED lamp can be approximated to Lambertian distribution [4] [5]. The specific Equation can be shown by Equation (1).

$$
I(\theta)=I_{0} \cos ^{m} \theta
$$

where $m$ is:

$$
m=\frac{-\ln 2}{\ln \left(\cos \theta_{1 / 2}\right)}
$$


In Equation (1) Equation (2), $I(\theta)$ is the illumination intensity in the direction of $\theta, I_{0}$ is the illumination intensity of viewing angle 0 on the LED axis; and $\theta_{1 / 2}$ is the angle of view of the light source when the light intensity is 0.5 times the axial light intensity $I_{0}$.

Photosynthetically active radiation (PAR) is an important parameter to study the effect of plants on light. It refers to the energy that can be absorbed and utilized by plants for photosynthesis in the process of light source radiation. It can be calculated by illuminance.

$$
\begin{array}{r}
I=\frac{\mathrm{d} \Phi}{\mathrm{d} \Omega} \\
E=\frac{\mathrm{d} \Phi}{\mathrm{d} S} \\
\mathrm{~d} \Omega=\frac{\mathrm{d} S}{R^{2}}
\end{array}
$$

In Equations (3)-(5), $\mathrm{d} \Phi$ is the luminous flux, $\mathrm{d} \Omega$ is the solid angle, and $\mathrm{d} S$ is the unit area illuminated on the object. $E$ is the illuminance, $R$ is the distance between the point to be measured and the light source.

Equation (6) can be obtained by combining Equation (1)-(5).

$$
E=\frac{I_{0} z^{m}}{\left(\left(x-x_{0}\right)^{2}+\left(x-x_{0}\right)^{2}+z^{2}\right)^{\frac{m+2}{2}}}
$$

where $x, y, z$ are relative coordinate values.

The three-dimensional distribution of illuminance of a single LED lamp is shown in "Figure 1".

\subsection{Lighting System Test of Light Source Group}

In order to give crops sufficient and uniform illumination, it is necessary to consider more reasonable arrangement of light sources. When the interference of light source is not considered, the illuminance of each light source can be linearized and added [6]. The light source distribution of agricultural greenhouse is shown in "Figure 2". Please do not revise any of the current designations.

Considering increasing the uniformity of illumination, the light source should be distributed symmetrically as far as possible. Based on the illumination uniformity of photovoltaic system, the lighting system test of light source group is established. The test system needs to meet the following conditions. The condition is shown in Equation (7).

$$
\left\{\begin{array}{l}
\mathrm{DU} \geq 75 \%, \mathrm{DU}=\frac{\text { average illumination rate of low quarter }}{\text { average illumination rate }} \times 100 \% \\
E(x, y) \geq 350 \text { lux }, E(x, y)=\sum_{i=1} E_{i}(x, y)
\end{array}\right.
$$

when the length and width of agricultural greenhouse is determined, the number of light sources required can be obtained by adjusting $N_{x}$ and $N_{y}$ (the number of light sources per row and column). At the same time, in order to reduce the waste 


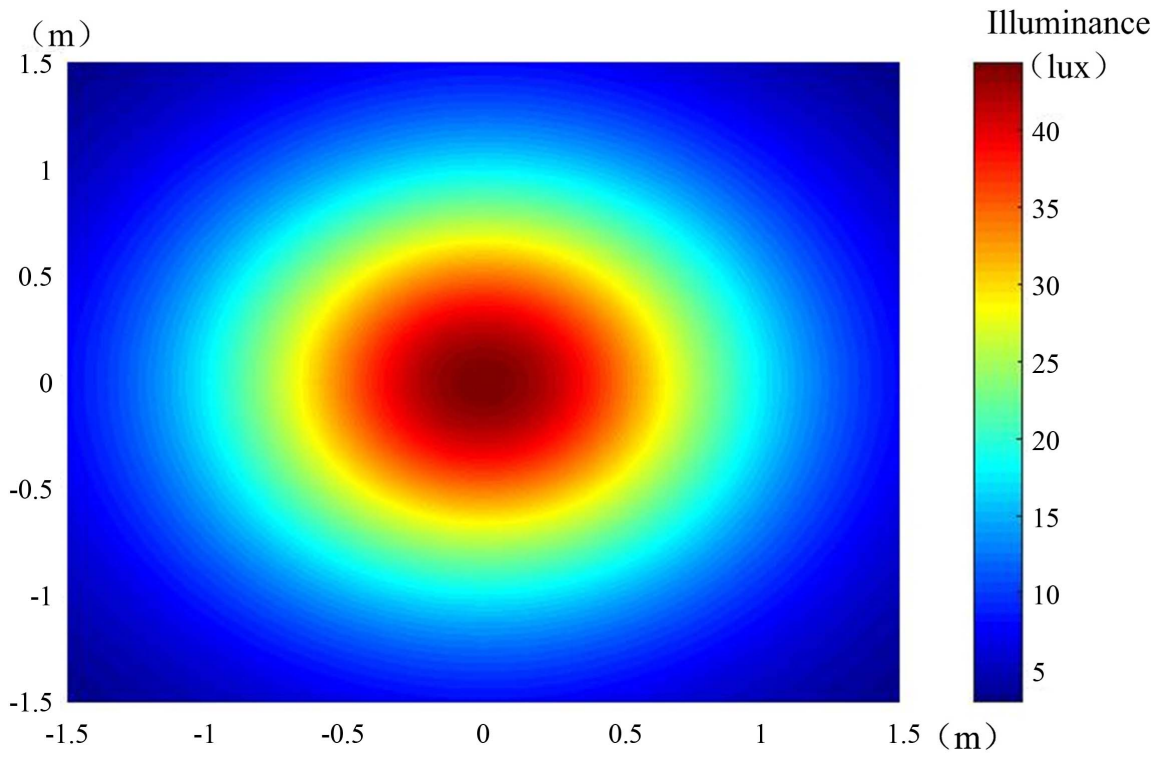

Figure 1. Illumination distribution of LED lamp.

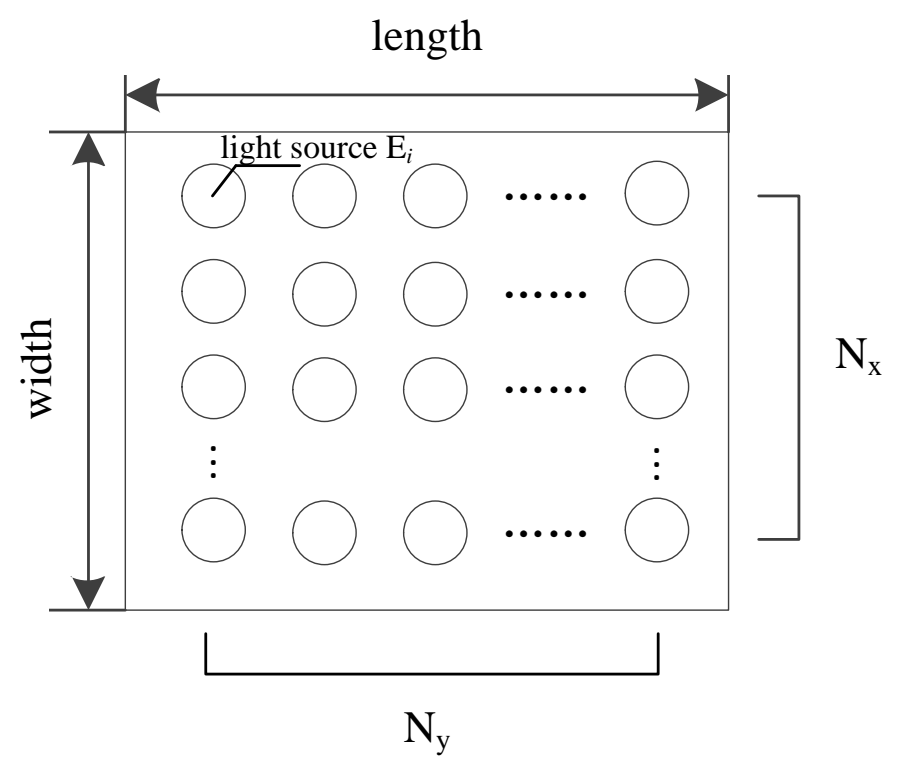

Figure 2. Distribution of light sources in agricultural greenhouses.

as much as possible, the number of light sources should be as small as possible. The objective function is shown in Equation (8).

$$
F=\min \left(N_{x} \times N_{y}\right)
$$

\subsection{Light Replenishment Scheduling Strategy Based on Light Source Group Displacement}

Now for the crop light supplement system, there are mobile light source groups available for use, and the illumination uniformity replenishment strategy can be completed by moving the light source [7]. The displacement position distribution of light source in agricultural greenhouse is shown in Figure 3. 


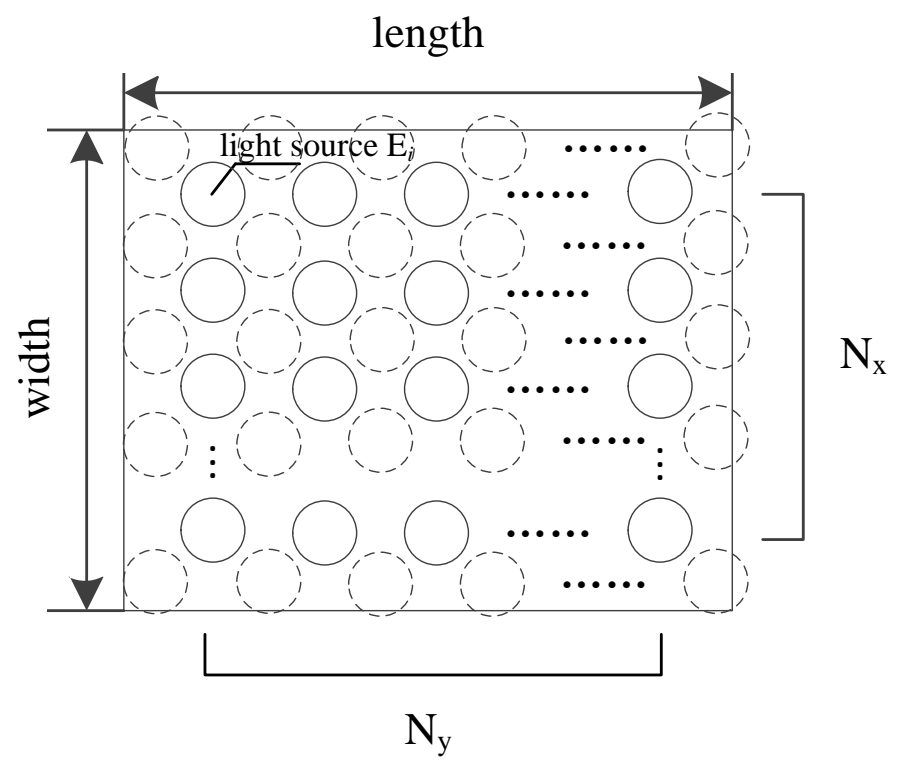

Figure 3. Distribution of light source displacement in agricultural greenhouse.

In "Figure 3", the dotted line is the position of the light source group after displacement. The solid line is the initial position of the light source. The Equation for calculating the position of the light source after displacement is shown in Equation (9).

$$
\left\{\begin{array}{l}
x^{\prime}=x+\Delta x \\
y^{\prime}=y+\Delta y
\end{array}\right.
$$

where $x^{\prime}$ and $y^{\prime}$ are the position of the light source after displacement; and are the displacement distance.

If Equation (9) is brought into $E(x, y)=\sum_{i=1} E_{i}(x, y), E^{\prime}$ can be obtained. The weighted average is calculated to get the $E_{\text {new }}$

$$
E_{\text {new }}=\alpha E^{\prime}+(1-\alpha) E_{\text {old }}
$$

In this paper, displacement algorithm is used to determine the position of light source every day. When the illumination uniformity is increased by adding light in a certain way, the iteration is saved and the light source position distribution within a week can be obtained.

\section{Experimental Result}

\subsection{Parameter Design}

This system is used to cover a $40 \mathrm{~m} \times 30 \mathrm{~m}$ agricultural greenhouse, and a scheme is worked out to minimize the time required for a farmer to maintain the irrigation system and maximize the uniformity of light source illumination. On the basis of a given agricultural greenhouse, try to determine the number and spacing of light sources, and establish a work schedule for finding a mobile pipeline, which also includes where to move the light source one day. 1000 lux per day shall not be less than any part of the farmland. 
This paper takes one day as the time scale and one week as the operation cycle to calculate the average illumination and illumination uniformity of agricultural greenhouse in a week. Compared with the situation of no moving light source, the advantages and disadvantages of crop light compensation scheduling strategy were evaluated.

\subsection{Experimental Results and Comparison}

- Control group experiment. This group does not carry out, the original way is used for crop light compensation. The average illumination distribution is shown in "Figure 4".

- Experimental group experiment. In this group of crop light compensation scheduling strategy, the original way is used for crop light compensation. The average illumination distribution is shown in "Figure 5".

- Experimental comparison (Table 1).

It can be seen that the performance of the experimental group is significantly better than that of the control group.

The light compensation strategy proposed in this paper has the following advantages:

- It can reduce the operation cost of crop light supplement system.

- It can improve the light uniformity in the crop greenhouse.

Table 1. Experimental comparison.

\begin{tabular}{ccc}
\hline Parameter & Minimum average illumination value (Lux) & DU \\
\hline Control group experiment & 351 & 77.9 \\
Experimental group experiment & 400 & 84.7 \\
\hline
\end{tabular}

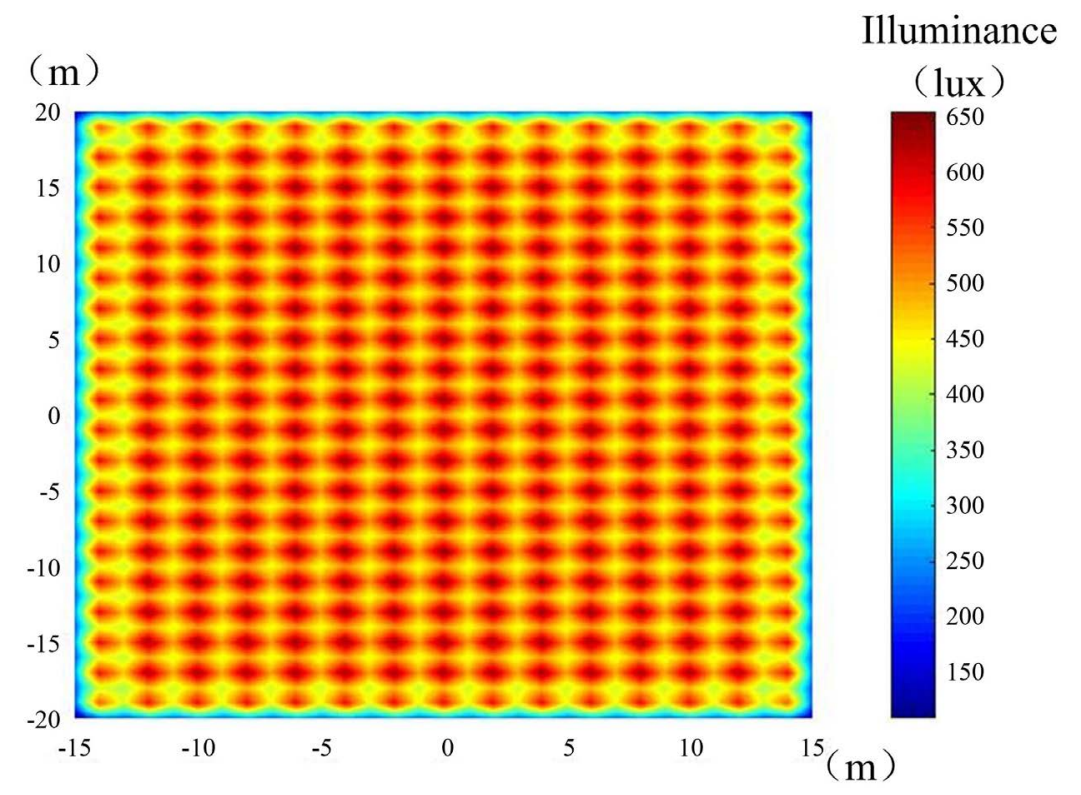

Figure 4. Illumination distribution map of agricultural greenhouse (control group experiment). 


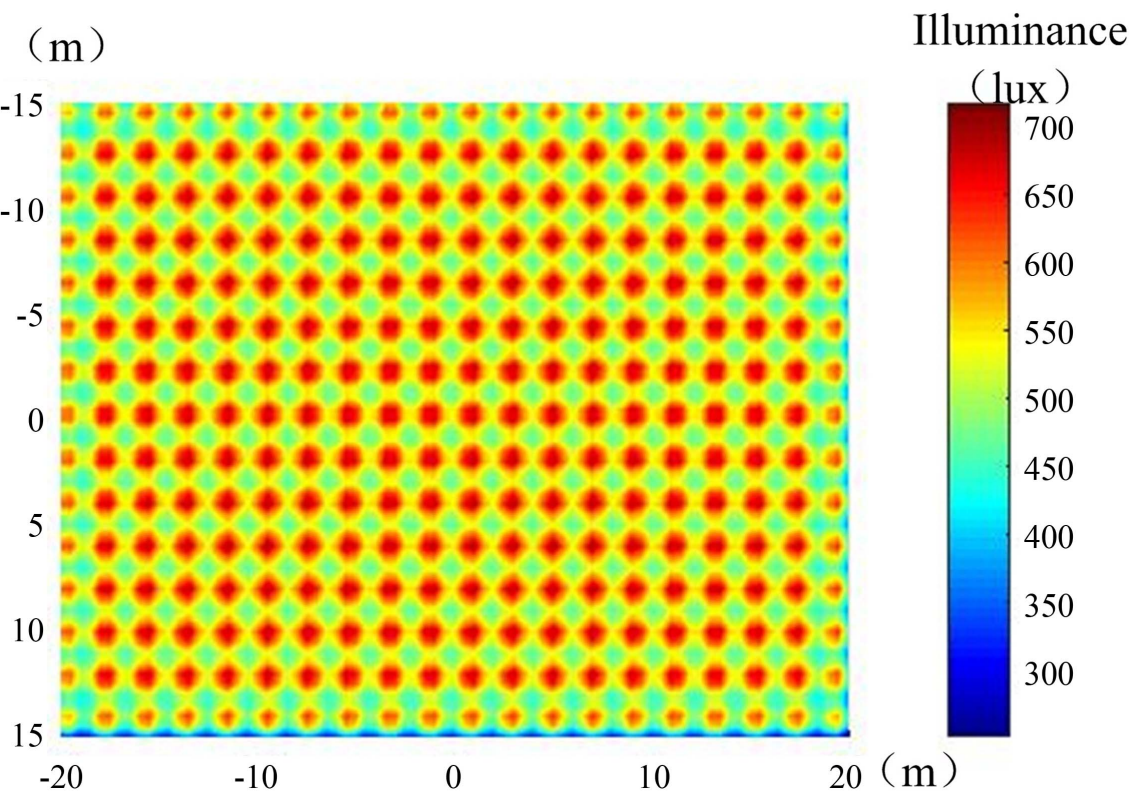

Figure 5. Illumination distribution map of agricultural greenhouse (experimental group experiment).

\section{Conclusion}

In order to solve the problem of supplementary light for crops in agricultural greenhouse, a scheduling strategy based on the displacement of light source group is proposed in this paper. This strategy can reduce the use of light source and reduce the cost, and improve the illumination uniformity in the greenhouse to improve the overall efficiency of the system. This has a positive significance for the growth of crops.

\section{Further Discussion}

Due to the different stages of plant growth, different spectral light sensitivity will be considered in the future work. At the same time, different light sources of crops often affect the effect of light supplement, which is also the place to be considered in the future. At the same time, different light sources of crops often affect the effect of light supplement, which is also the place to be considered in the future. Therefore, in the future, our group intends to make further research by combining the light and illumination of different spectra with $A$ scheduling strategy of light supplement in agricultural greenhouse based on the displacement of light source group proposed in this paper.

\section{Conflicts of Interest}

The authors declare no conflicts of interest regarding the publication of this paper.

\section{References}

[1] (2017) Agriculture LED Lighting: Improving sustainable Agriculture. 
https://www.arrow.com/zh-cn/research-and-events/articles/agriculture-lighting

[2] Folta, K.M. and Maruhnich, S.A. (2007) Green Light-A Signal to Slowdown or Stop. Journal of Experimental Botany, 58, 3099-3111. https://doi.org/10.1093/jxb/erm130

[3] Bula, R.J., Morrow, R.C., Tibbitts, T.W., Ignatius, R.W., Martin, T.S., et al. (1991) Light-Emitting Diodes as a Radiation Source for Plants. HortScience, 26, 203-205. https://doi.org/10.21273/HORTSCI.26.2.203

[4] Zhu, Z. (2016) Design of Lighting Control Device for Plants Based on Research of Illumination Distribution Characteristic of LED Source. Fujian Agriculture and Forestry University, Fuzhou.

[5] Tang, H.-Z., Wen, S.-S., Fu, M., He, G., Zhang, H.-Y., Liao, S.-X. and Kang, L.-J. (2019) Design of LED Plant Lighting Source Based on Particle Swarm Optimization Algorithm under Photons System. Chinese Journal of Luminescence, 40, 340-348. https://doi.org/10.3788/fgxb20194003.0340

[6] Jin, J. (2012) Research on Agriculture Greenhouse Ccontrol System Based on hybrid energy. Yanbian University, Yanji.

[7] Hu, S.Q. (2019) Effects of Different Durations of Illumination and Supplemental Light Quality on Growth of Oriental Lily. Journal of Zhejiang Agricultural Sciences, $60,469-472+475$. 